\title{
Photometric observations of an extreme mass ratio overcontact binary
}

\author{
X. Zhou ${ }^{1,2,3,4}$, B. Soonthornthum ${ }^{2}$ and S.-B. Qian ${ }^{1,3,4,5}$ \\ 1 Yunnan Observatories, Chinese Academy of Sciences (CAS), P.O. Box 110, \\ 650216 Kunming, China, (E-mail: zhouxiaophy@ynao.ac.cn) \\ 2 National Astronomical Research Institute of Thailand, 260 Moo 4, T. \\ Donkaew, A. Maerim, Chiangmai, 50180, Thailand \\ ${ }^{3}$ Key Laboratory of the Structure and Evolution of Celestial Objects, Chinese \\ Academy of Sciences, P. O. Box 110, 650216 Kunming, China \\ 4 Center for Astronomical Mega-Science, Chinese Academy of Sciences, $20 \mathrm{~A}$ \\ Datun Road, Chaoyang District, Beijing, 100012, China \\ 5 University of the Chinese Academy of Sciences, Yuquan Road 19\#, \\ Sijingshang Block, 100049 Beijing, P. R. China
}

Received: October 30, 2019; Accepted: January 23, 2020

\begin{abstract}
Our $R_{C}$ and $I_{C}$ light curves of CSS J135012.1+272259 were analyzed with the Wilson-Devinney (W-D) program. The results reveal that CSS $\mathrm{J} 135012.1+272259$ is an extreme mass ratio overcontact binary with mass ratio $q=0.147$. It may be in the final evolutionary stage of cool short-period binaries and merge into a single rapid-rotation star to form a blue straggler or FK Com type star. Also, 25 extreme mass ratio overcontact binary systems $(q \leq 0.15)$ are collected for long-term monitoring. These targets will improve understanding of the pre-outburst state of overcontact binaries and enrich knowledge of the merger mechanism.
\end{abstract}

Key words: binaries: eclipsing - binaries: photometry - light curves - stellar mergers

\section{Introduction}

Stellar mergers are estimated to be common events in the Galaxy. Theoretical models predict that an overcontact (OC) binary system will merge when its mass ratio reaches $q \approx 0.07-0.09$ (Arbutina, 2012). Only a handful of these transients have been noted in the Milky Way: V4332 Sgr (Hayashi et al., 1994), V838 Mon (Brown et al., 2002), V1309 Sco (Nakano et al., 2008), and OGLE-2002-BLG-360 (Tylenda et al., 2013). The red nova V1309 Sco is the best studied stellar merger case to date. The discovery of V1309 Sco (Nova Scorpii 2008 ) and the fact that its progenitor is an extreme mass ratio OC system with a rapidly decreasing orbital period triggered our interest on extreme mass ratio OC systems. Photometric and spectrometric data on OC binaries have been accumulating rapidly over the past few decades, owing to large sky survey projects 
such as the Optical Gravitational Lensing Experiment (Rucinski, 1997), the All Sky Automated Survey (Jayasinghe et al., 2019), the Large Sky Area MultiObject Fibre Spectroscopic Telescope (Qian et al., 2019) and the Gaia mission (Gaia Collaboration et al., 2016). Also, many extreme mass ratio OC systems or potential stellar merger candidates have been reported.

\section{Photometric investigations of CSS J135012.1+272259}

CSS J135012.1+272259 is a short period OC system. $R_{C}$ and $I_{C}$ light curves were obtained on March 8th, 2018 with the 2.4-m telescope at Thai National Observatory, National Astronomical Research Institute of Thailand (Soonthornthum, 2018). The ephemeris is:

$$
\operatorname{Min} . \mathrm{I}(\mathrm{HJD})=2458186.4009+0.232465 \times E \text {. }
$$

The effective temperature is $6137 \mathrm{~K}$, as given by the LAMOST survey. The Wilson-Devinney program (Wilson \& Devinney, 1971; Van Hamme \& Wilson, 2007; Wilson \& Van Hamme, 2014) of version 2013 was applied to model the light curves, with solutions in Table 1. A q-search diagram and light curves are in Fig. 1.

Table 1. CSS J135012.1+272259 Phorometric Solutions

\begin{tabular}{|c|c|c|}
\hline Parameters & $\begin{array}{l}\text { Values } \\
\text { without } l_{3}\end{array}$ & $\begin{array}{l}\text { Values } \\
\text { with } l_{3}\end{array}$ \\
\hline$\overline{T_{1}(K)}$ & 6137 (fixed) & 6137 (fixed) \\
\hline $\mathrm{q}\left(M_{2} / M_{1}\right)$ & $0.114( \pm 0.004)$ & $0.147( \pm 0.011)$ \\
\hline$i\left(0^{\circ}\right)$ & $75.9( \pm 1.3)$ & $79.1( \pm 1.6)$ \\
\hline$\Omega_{1}=\Omega_{2}$ & $1.98( \pm 0.01)$ & $2.06( \pm 0.03)$ \\
\hline$T_{2}(K)$ & $5838( \pm 43)$ & $5891( \pm 55)$ \\
\hline$\Delta T(K)$ & 299 & 246 \\
\hline$T_{2} / T_{1}$ & $0.951( \pm 0.007)$ & $0.960( \pm 0.009)$ \\
\hline$L_{1} /\left(L_{1}+L_{2}\right)\left(R_{c}\right)$ & $0.8931( \pm 0.0004)$ & $0.863( \pm 0.009)$ \\
\hline$L_{1} /\left(L_{1}+L_{2}\right)\left(I_{c}\right)$ & $0.8905( \pm 0.0004)$ & $0.860( \pm 0.009)$ \\
\hline$L_{1} /\left(L_{1}+L_{2}+L_{3}\right)\left(R_{c}\right)$ & & $0.705( \pm 0.036)$ \\
\hline$L_{1} /\left(L_{1}+L_{2}+L_{3}\right)\left(I_{c}\right)$ & & $0.702( \pm 0.038)$ \\
\hline$L_{3} /\left(L_{1}+L_{2}+L_{3}\right)\left(R_{c}\right)$ & & $0.183( \pm 0.039)$ \\
\hline$L_{3} /\left(L_{1}+L_{2}+L_{3}\right)\left(I_{c}\right)$ & & $0.184( \pm 0.041)$ \\
\hline$r_{1}($ pole $)$ & $0.530( \pm 0.003)$ & $0.518( \pm 0.005)$ \\
\hline$r_{1}($ side $)$ & $0.590( \pm 0.005)$ & $0.572( \pm 0.008)$ \\
\hline$r_{1}($ back $)$ & $0.610( \pm 0.005)$ & $0.596( \pm 0.008)$ \\
\hline$r_{2}($ pole $)$ & $0.202( \pm 0.015)$ & $0.223( \pm 0.032)$ \\
\hline$r_{2}($ side $)$ & $0.211( \pm 0.018)$ & $0.234( \pm 0.039)$ \\
\hline$r_{2}(b a c k)$ & $0.247( \pm 0.040)$ & $0.278( \pm 0.092)$ \\
\hline$f$ & $18.6 \%( \pm 20.1 \%)$ & $34.0 \%( \pm 30.8 \%)$ \\
\hline$\Sigma \omega(O-C)^{2}$ & 0.0051 & 0.0045 \\
\hline
\end{tabular}



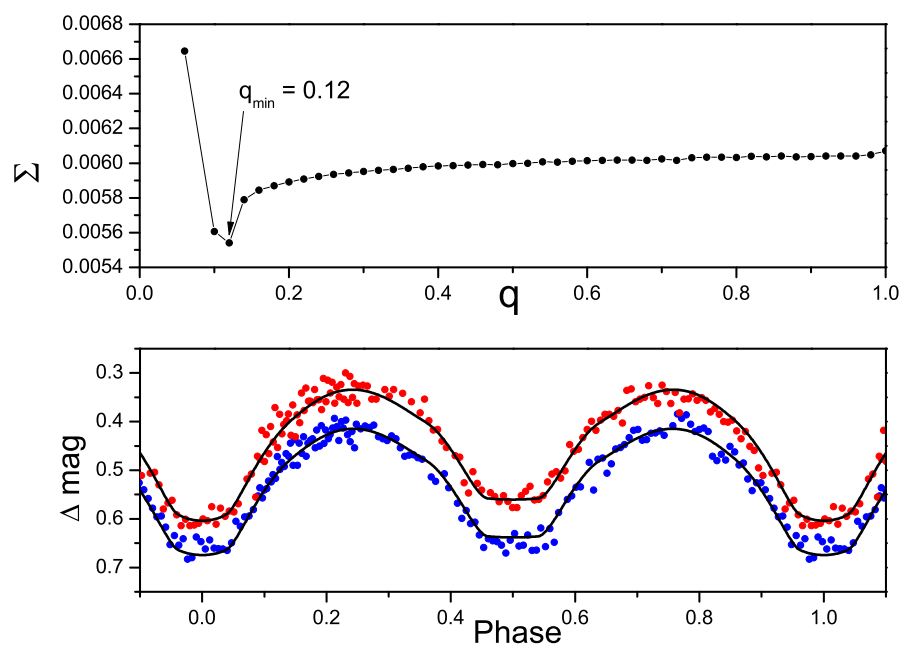

Figure 1. In the upper panel, the minimum mass ratio is determined to be $q_{\min }=0.12$ with the q-search method. In the lower panel, the red and blue circles are $R_{C}$ and $I_{C}$ light curves, respectively. The black lines are theoretical light curves.

\section{Discussion and conclusions}

Photometric solutions show that CSS J135012.1+272259 is an A-subtype extreme mass ratio $\mathrm{OC}$ binary with mass ratio $q=0.147$ that is predicted to merge and make a blue straggler or FK Com type star in the center (Tylenda et al., 2011; Ferreira et al., 2019). A-type is a sub-type of the W UMa's, and the W UMa's are a sub-type of the OCs (Binnendijk, 1970). More observations of extreme mass ratio OC systems are needed since only a few stellar merger events have been reported and there are some discrepancies between observed features and theoretical models. Thus, 25 targets with mass ratio $q \leq 0.15$ have been collected: V857 Her, ASAS J083241+2332.4, SX Crv, V53 (a member of the Globular Cluster M4), AW UMa, ZZ Ps, V870 Ara, AW CrB, DN Boo, ASAS J082243+1927, V1191 Cyg, CK Boo, GR Vir, FG Hya, AL Lep, V776 Cas, V345 Gem, V410 Aur, V710 Mon, DZ Psc, HV Aqr, CSS J135012.1+272259, XY LMi, EM Psc, and TYC 4157-683-1. All of these targets will be monitored long-term.

Acknowledgements. This research was supported by the National Natural Science Foundation of China (Grant No. 11703080 and 11703082), the Joint Research Fund in Astronomy (Grant No. U1931101) under cooperative agreement between the National Natural Science Foundation of China and Chinese Academy of Sciences, and the Yunnan Natural Science Foundation (Grant No. 2018FB006). It was part of the research activities at the National Astronomical Research Institute of Thailand (Public Organization). 


\section{References}

Arbutina, B., The Minimum Mass Ratio for Contact Close Binary Systems of W Ursae Majoris-Type. 2012, Publications de l'Observatoire Astronomique de Beograd, 91, 391

Binnendijk, L., The orbital elements of W Ursae Majoris systems. 1970, Vistas in Astronomy, 12, 217, DOI: 10.1016/0083-6656(70)90041-3

Brown, N. J., Waagen, E. O., Scovil, C., et al., Peculiar variable in Monoceros. 2002, IAU Circ., 7785, 1

Ferreira, T., Saito, R. K., Minniti, D., et al., The asymptotic evolution of the stellar merger V1309 Sco: a Blue Straggler in the making? 2019, Mon. Not. R. Astron. Soc., 486, 1220, DOI: 10.1093/mnras/stz878

Gaia Collaboration, Prusti, T., de Bruijne, J. H. J., et al., The Gaia mission. 2016, Astron. Astrophys., 595, A1, DOI: 10.1051/0004-6361/201629272

Hayashi, S. S., Yamamoto, M., \& Hirosawa, K., Nova Sagittarii 1994. 1994, IAU Circ., 5942, 1

Jayasinghe, T., Stanek, K. Z., Kochanek, C. S., et al., The ASAS-SN catalogue of variable stars - II. Uniform classification of 412000 known variables. 2019, Mon. Not. R. Astron. Soc., 486, 1907, DOI: 10.1093/mnras/stz844

Nakano, S., Nishiyama, K., Kabashima, F., \& Sakurai, Y., Possible Nova in Scorpius. 2008, Central Bureau Electronic Telegrams, 1496, 1

Qian, S.-B., Shi, X.-D., Zhu, L.-Y., et al., More than two hundred and fifty thousand spectroscopic binary or variable star candidates discovered by LAMOST. 2019, Research in Astronomy and Astrophysics, 19, 064, DOI: 10.1088/1674-4527/19/5/64

Rucinski, S. M., Eclipsing Binaries in the OGLE Variable Star Catalog.II.Light Curves of the W UMA-Type Systems in Baade's Window. 1997, Astron. J., 113, 1112, DOI: $10.1086 / 118329$

Soonthornthum, B., Present and future of astronomy in Thailand. 2018, Nature Astronomy, 2, 355, DOI: 10.1038/s41550-018-0464-6

Tylenda, R., Hajduk, M., Kamiński, T., et al., V1309 Scorpii: merger of a contact binary. 2011, Astron. Astrophys., 528, A114, DOI: 10.1051/0004-6361/201016221

Tylenda, R., Kamiński, T., Udalski, A., et al., OGLE-2002-BLG-360: from a gravitational microlensing candidate to an overlooked red transient. 2013, Astron. Astrophys., 555, A16, DOI: 10.1051/0004-6361/201321647

Van Hamme, W. \& Wilson, R. E., Third-Body Parameters from Whole Light and Velocity Curves. 2007, Astrophys. J., 661, 1129, DOI: 10.1086/517870

Wilson, R. E. \& Devinney, E. J., Realization of Accurate Close-Binary Light Curves: Application to MR Cygni. 1971, Astrophys. J., 166, 605, DOI: 10.1086/150986

Wilson, R. E. \& Van Hamme, W., Unification of Binary Star Ephemeris Solutions. 2014, Astrophys. J., 780, 151, DOI: 10.1088/0004-637X/780/2/151 